\title{
STRESS STATE OF PLATE WITH INCISIONS UNDER THE ACTION OF OSCILLATING CONCENTRATED FORCES
}

\author{
Vasyl' SHVABYUK*, Heorhiy SULYM*, Olena MIKULICH* \\ *Department of Technical Mechanic, Lutsk National Technical University, 75 Lvivska st., Lutsk, 43018, Ukraine \\ **Bialystok University of Technology, ul. Wiejska 45C, 15-351 Bialystok, Poland \\ shvabyk@lutsk-ntu.com.ua, h.sulym@pb.edu.pl, shypra@ukr.net
}

received 2 March 2015, revised 9 October 2015, accepted 14 October 2015

\begin{abstract}
This paper proposes the novel technique for analysis of dynamic stress state of multi-connected infinite plates under the action of oscillating forces. Calculation of dynamic stresses at the incisions of plates is held using the boundary-integral equation method and the theory of complex variable functions. The numerical implementation of the developed algorithmis based on the method of mechanical quadratures and collocation technique. The algorithm is effective in the analysis of the stress state caused by steady-state vibrations of plates.
\end{abstract}

Key words: Stress State, Plates, Incision, Hole

\section{INTRODUCTION}

Many structural elements, which are used in modern engineering, can be modelled as plates during the structural analysis. Frequently these elements operate under the dynamic loads. Thus, estimation of their strength is based on the calculation of dynamic stress state near defects, which can be present in the plate. This greatly complicates the stress state due to the reflection of elastic waves from the defects' boundary inside the plate.

In contrast with static loading, the strength of structural elements depends on the frequency of the applied dynamic load.

Methods for analysis of the stress state of structural elements with one or more holes under dynamic loadings were developed in the works: Brebbia et al. (1984), Guz et al. (1978), Savin (1968), Timoshenko (1967), Pao and Mow (1971), Mow and Mente (1963), etc.

The problem of diffraction of elastic waves in an infinite plate with a circular hole or a system circular holes was solved by Guz et al. (1978), Pao et al. (1971) and Mow et al. (1963). In the works by Kubenko (1967) and Guz et al. (1978) the problem of the concentration of dynamic stress near holes of non-canonical form is studied by the method, which is based on the method of series and the boundary shape perturbation technique.

In the works Mushelishvili (1966) and Panasyuk et al. (1984) an algorithm for studying of the stress state of plates of different shape under the static loadings is developed. This algorithm is based on the boundary integral equation method and the theory of a complex variable.

The main advantage of this approach is its universality and high accuracy in the case of multiply connected plates of difficult shape or infinite plates with holes, which are under the action of concentrated forces.

Systems of integral equations for determination of the dynamic stress state of plates are derived in the works: Kupradze (1963),
Sherman (1962), Sladek et al. (2000). Numerical analysis of the stress state is held by the boundary element method in the works Benerjee (1994) and Brebbia et al. (1984). The Somigliana type integral formula is used. Thus, integral equations for relative displacements are directly obtained. Stress at the boundary is determined by the numerical differentiation. At high frequencies, numerical differentiation can lead to significant errors, thus, the technique which utilize stress integral formulae for dynamic problems is of high importance.

\section{SOLUTION OF THE PROBLEM}

\subsection{Statement of the problem}

Consider an infinite plate with incision that is under the influence of concentrated oscillating forces $Q_{1} e^{i \omega \tau}, Q_{2} e^{i \omega \tau}$, where $\omega$ is the frequency of the applied loading, and $\tau$ is time (Fig. 1). The problem consists in determination of the dynamic stresses at the boundary incision in the plate.

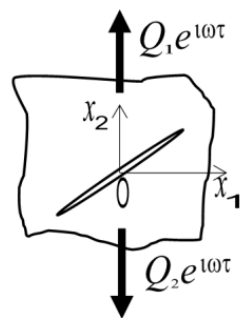

Fig. 1. Model of the plate

The center of gravity of the plate is placed at the origin of a Cartesian coordinate system $O x_{1} x_{2}$. Symbol $D$ denotes the 
domain occupied by the plate, and $L$ is the boundary of the domain $D$.

\subsection{Integral equation of the problem}

For the plane stress state the potential solution for image motion is selected as (Bonnet, 1995):

$u_{j}=\int_{L} p_{i} U_{i j}^{*} d s+\int_{D} Q_{i} U_{i j}^{*} d D$,

where $p_{1}, p_{2}$ are unknown complex potential function.

The representation of the image $U_{i j}^{*}$ should be choosing with the regard to Zommerfeld conditions, since the plate is infinite. It has the form (Brebbia et al., 1984):

$U_{i j}^{*}=\frac{1}{2 \pi \rho c_{2}^{2}}\left(\psi \delta_{i j}-\chi \cdot r_{i} r_{j}\right)$,

where

$\psi=\frac{i \pi}{2}\left(-H_{0}^{2}\left(\frac{\omega r}{c_{2}}\right)+\frac{c_{2}}{\omega r}\left(H_{1}^{2}\left(\frac{\omega r}{c_{2}}\right)-\frac{c_{2}}{c_{1}} H_{1}^{2}\left(\frac{\omega r}{c_{1}}\right)\right)\right)$,

$\chi=\frac{i \pi}{2}\left(H_{2}^{2}\left(\frac{\omega r}{c_{2}}\right)-\left(\frac{c_{2}}{c_{1}}\right)^{2} H_{2}^{2}\left(\frac{\omega r}{c_{1}}\right)\right)$,

$r_{i}=\frac{\partial r}{\partial x_{i}}, \quad r=\sqrt{\left(x_{1}-x_{1}^{0}\right)^{2}+\left(x_{2}-x_{2}^{0}\right)^{2}}, \quad c_{1}, c_{2}$ are the speeds of expansion and shear waves: $c_{1}^{2}=\frac{E}{\rho\left(1-v^{2}\right)}, c_{2}^{2}=$ $\frac{E}{2 \rho(1+v)}, E$ is the Young's modulus, $\rho$ is the density of the material; $v$ is the Poisson ratio; $\mathrm{H}_{\mathrm{k}}^{2}(\mathrm{r})=\mathrm{J}_{\mathrm{k}}(\mathrm{r})-\mathrm{Y}_{\mathrm{k}}(\mathrm{r})$ are Hankel functions of the second kind; $J_{k}(r), Y_{k}(r)$ are Bessel functions of the first and second kinds $(i, j=1,2)$. The integration over the domain and along the boundary is performed within variable $x_{1}^{0}, x_{2}^{0}$. Here the time factor $e^{i \omega \tau}$ is omitted at the displacements and the stresses terms.

Determination of the stresses at an arbitrary point of the plate with normal $\vec{n}$ are performed by the formula (Savin, 1968):

$$
\begin{aligned}
& 2\left(\sigma_{n}-i \tau_{n}\right)=\frac{2 E}{1-v} \operatorname{Re}\left(\frac{\partial}{\partial \bar{z}}\left(u_{1}-i u_{2}\right)\right)+ \\
& +e^{2 i \alpha} \frac{2 E}{1+v}\left(\frac{\partial}{\partial z}\left(u_{1}-i u_{2}\right)\right),
\end{aligned}
$$

where $\alpha$ is the angle between the normal to the boundary of the plate and the axis $0 \mathrm{x}_{1} ; \frac{\partial}{\partial \overline{\mathrm{z}}}=\frac{1}{2}\left(\left(\frac{\partial}{\partial \mathrm{x}_{1}}-\mathrm{i} \frac{\partial}{\partial \mathrm{x}_{2}}\right)\right), \frac{\partial}{\partial \mathrm{z}}=$ $\frac{1}{2}\left(\left(\frac{\partial}{\partial x_{1}}+i \frac{\partial}{\partial x_{2}}\right)\right)$.

Since the functions $U_{i j}^{*}$ along with the displacement $u_{j}$ are complex, the determination of the stress is performed for the real and imaginary parts of images (1):

$u_{j}^{R}=\int_{L}\left(p_{i}^{R} U_{i j}^{*}-p_{i}^{I} U_{i j}^{* I}\right) d s+\int_{D}\left(Q_{i}^{R} U_{i j}^{*}-Q_{i}^{I} U_{i j}^{* I}\right) d D$,

$u_{j}^{I}=\int_{L}\left(p_{i}^{I} U_{i j}^{* R}+p_{i}^{R} U_{i j}^{* I}\right) d s+\int_{D}\left(Q_{i}^{I} U_{i j}^{*}+Q_{i}^{R} U_{i j}^{* I}\right) d D$,

where the values with the superscript $R$ are real parts of corresponding functions $F_{k}^{R}=\operatorname{Re}\left(F_{k}\right)$, and the values with the superscript $I$ are the imaginary parts of corresponding functions $F_{k}^{I}=\operatorname{Im}\left(F_{k}\right), k=1,2$.

Substituting the representation for displacement in the formula (2), we obtain formulas for determining stresses at the boundary of the plate:
$2\left(\sigma_{n}^{R}-i \tau_{s n}^{R}\right)=\int_{L}\left(f_{1}^{R} q^{R}-f_{1}^{I} q^{I}\right) d t+\int_{L}\left(f_{2}^{R} \bar{q}^{R}-f_{2}^{I} \bar{q}^{I}\right) d \bar{t}$ $\int_{D}\left(f_{1}^{R} Q^{R}-f_{1}^{I} Q^{I}\right) d D+\int_{L}\left(f_{2}^{R} \bar{Q}^{R}-f_{2}^{I} \bar{Q}^{I}\right) d D$

$2\left(\sigma_{n}^{I}-i \tau_{s n}^{I}\right)=\int_{L}\left(f_{1}^{R} q^{I}+f_{1}^{I} q^{R}\right) d t+\int_{L}\left(f_{2}^{R} \bar{q}^{I}+f_{2}^{I} \bar{q}^{R}\right) d \bar{t}$ $+\int_{D}\left(f_{1}^{R} Q^{I}+f_{1}^{I} Q^{R}\right) d D+\int_{L}\left(f_{2}^{R} \bar{Q}^{I}+f_{2}^{I} \bar{Q}^{R}\right) d D$,

where $f_{k}^{R}=f_{k}^{R}\left(x_{1}, x_{2}, x_{1}^{0}, x_{2}^{0}\right), f_{k}^{I}=f_{k}^{I}\left(x_{1}, x_{2}, x_{1}^{0}, x_{2}^{0}\right)$, $k=1,2$ are functions of the real argument, which contain Bessel functions of second and first kind respectively and are obtained similarly to those in Mikulich and Maksymovych (2011); $q^{R}=\frac{i p^{R} d s}{d t}, q^{I}=\frac{i p^{I} d s}{d t}$ are unknown functions to be determined, $t=x_{1}^{0}+i x_{2}^{0}$.

Integration of functions $f_{k}^{R}, k=1,2$ for small values of the argument leads to singularity. To establish their characteristics we use the asymptotic expressions for the Bessel functions of the second kind for small values of the argument (Elbert and Laforgia, 1986). Then the formula for determining of the stresses can be written as:

$2\left(\sigma_{n}^{R}-i \tau_{s n}^{R}\right)=\frac{1}{2 \pi i} \frac{1+v}{2} \int_{L}\left(\left(\frac{d z}{d \bar{z}} \frac{\bar{z}-\bar{t}}{z-t}-1\right) \frac{1}{z-t}\right) q^{R} d t$

$-\frac{1}{2 \pi i} \int_{L}\left(-\frac{1+v}{2} \frac{1}{\bar{z}-\bar{t}}+\frac{d z}{d \bar{z}} \frac{3-v}{2} \frac{1}{z-t}\right) \bar{q}^{R} d \bar{t}+$

$\int_{L}\left(G_{1}^{R} q^{R}-f_{1}^{I} q^{I}\right) d t+\int_{L}\left(G_{2}^{R} \bar{q}^{R}-f_{2}^{I} \bar{q}^{I}\right) d \bar{t}+$

$\int_{D}\left(f_{1}^{R} Q^{R}-f_{1}^{I} Q^{I}\right) d D+\int_{D}\left(f_{2}^{R} \bar{Q}^{R}-f_{2}^{I} \bar{Q}^{I}\right) d D ;$

$2\left(\sigma_{n}^{I}-i \tau_{s n}^{I}\right)=\frac{1}{2 \pi i} \frac{1+v}{2} \int_{L}\left(\left(\frac{d z}{d \bar{z}} \frac{\bar{z}-\bar{t}}{z-t}-1\right) \frac{1}{z-t}\right) q^{I} d t$

$-\frac{1}{2 \pi i} \int_{L}\left(-\frac{1+v}{2} \frac{1}{\bar{z}-\bar{t}}+\frac{d z}{d \bar{z}} \frac{3-v}{2} \frac{1}{z-t}\right) \bar{q}^{I} d \bar{t}+$

$\int_{L}\left(G_{1}^{R} q^{I}+f_{1}^{I} q^{R}\right) d t+\int_{L}\left(G_{2}^{R} \bar{q}^{I}+f_{2}^{I} \bar{q}^{R}\right) d \bar{t}+$

$\int_{D}\left(f_{1}^{R} Q^{I}+f_{1}^{I} Q^{R}\right) d D+\int_{D}\left(f_{2}^{R} \bar{Q}^{I}+f_{2}^{I} \bar{Q}^{R}\right) d D ;$

where $f_{k}^{I}, G_{k}^{R}=G_{k}^{R}\left(x_{1}, x_{2}, x_{1}^{0}, x_{2}^{0}\right), k=1,2$ are bounded and continuous functions of real argument everywhere in $D$.

Let us perform the limiting transition when $\left(x_{1}, x_{2}\right) \rightarrow L$ in the last formula according to Plemelj-Sokhotski formulas (Savin, 1968). Consequently, integral equations for determination of the unknown functions $q^{R}$ and $q^{I}$ for given loading at the boundary are obtain:

$\overline{\mathrm{q}}^{\mathrm{R}}+\frac{1}{2 \pi \mathrm{i}} \frac{1+v}{2} \int_{\mathrm{L}}\left(\left(\frac{\mathrm{dz}}{\mathrm{d} \overline{\mathrm{z}}} \frac{\overline{\mathrm{z}}-\overline{\mathrm{t}}}{\mathrm{z}-\mathrm{t}}-1\right) \frac{1}{\mathrm{z}-\mathrm{t}}\right) \mathrm{q}^{\mathrm{R}} \mathrm{dt}$

$-\frac{1}{2 \pi i} \int_{L}\left(-\frac{1+v}{2} \frac{1}{\bar{z}-\bar{t}}+\frac{d z}{d \bar{z}} \frac{3-v}{2} \frac{1}{z-t}\right) \bar{q}^{R} d \bar{t}+$

$\int_{L}\left(G_{1}^{R} q^{R}-f_{1}^{I} q^{I}\right) d t+\int_{L}\left(G_{2}^{R} \bar{q}^{R}-f_{2}^{I} \bar{q}^{I}\right) d \bar{t}=2 S^{R} ;$

$\bar{q}^{I}+\frac{1}{2 \pi i} \frac{1+v}{2} \int_{L}\left(\left(\frac{d z}{d \bar{z}} \frac{\bar{z}-\bar{t}}{z-t}-1\right) \frac{1}{z-t}\right) q^{I} d t$

$\frac{1}{2 \pi i} \int_{L}\left(-\frac{1+v}{2} \frac{1}{\bar{z}-\bar{t}}+\frac{d z}{d \bar{z}} \frac{3-v}{2} \frac{1}{z-t}\right) \bar{q}^{I} d \bar{t}+$

$\int_{L}\left(G_{1}^{R} q^{I}+f_{1}^{I} q^{R}\right) d t+\int_{L}\left(G_{2}^{R} \bar{q}^{I}+f_{2}^{I} \bar{q}^{R}\right) d \bar{t}=2 S^{I}$,

where the first and second integrals are evaluated for their Cauchy principal value; $S^{R}, S^{I}$ are known function:

$2 S^{R}=\int_{D}\left(f_{1}^{I} Q^{I}-f_{1}^{R} Q^{R}\right) d D+\int_{D}\left(f_{2}^{I} \bar{Q}^{I}-f_{2}^{R} \bar{Q}^{R}\right) d D$,

$2 S^{I}=\int_{D}\left(f_{1}^{R} Q^{I}+f_{1}^{I} Q^{R}\right) d D+\int_{D}\left(f_{2}^{R} \bar{Q}^{I}+f_{2}^{I} \bar{Q}^{R}\right) d D$.

Stresses at the boundary of the incisions of the plate are determined in the absence of the contact of the incision's boundary. This is verified by the formulas: 
$u_{1}^{R}-i u_{2}^{R}=-\frac{1+v}{\pi E i} \int_{L}\left(F_{1}^{R} q^{R}-F_{1}^{I} q^{I}\right) d t+$

$\int_{L}\left(F_{2}^{R} \bar{q}^{R}-F_{2}^{I} \bar{q}^{I}\right) d \bar{t}+\int_{D}\left(F_{1}^{R} Q^{R}-F_{1}^{I} Q^{I}\right) d D+$

$\int_{L}\left(f_{2}^{R} \bar{Q}^{R}-F_{2}^{I} \bar{Q}^{I}\right) d D$

$u_{1}^{I}-i u_{2}^{I}=-\frac{1+v}{\pi E i} \int_{L}\left(F_{1}^{R} q^{I}+F_{1}^{I} q^{R}\right) d t+$

$\int_{L}\left(F_{2}^{R} \bar{q}^{I}+F_{2}^{I} \bar{q}^{R}\right) d \bar{t}+\int_{D}\left(F_{1}^{R} Q^{I}+F_{1}^{I} Q^{R}\right) d D+$

$\int_{L}\left(f_{2}^{R} \bar{Q}^{I}+F_{2}^{I} \bar{Q}^{R}\right) d D$

where $F_{k}^{R}=F_{k}^{R}\left(x_{1}, x_{2}, x_{1}^{0}, x_{2}^{0}\right), F_{k}^{I}=F_{k}^{I}\left(x_{1}, x_{2}, x_{1}^{0}, x_{2}^{0}\right)$, $k=1,2$ are functions of the real argument, which contain Bessel functions of second and first kind respectively and are obtained similar to those by Mikulich (2012).

\subsection{Numeric solution of the algortim}

To study the stress state of the infinite plate with one incision denote its boundary contour as $\Omega$. The incision in the plates is modeled as an elliptic hole with axis ratio of 10 . Its equation in parametric form is as follows: $x_{1}=\varphi(\theta), x_{2}=\psi(\theta), 0<$ $\theta<2 \pi$. Parameter $\theta$ is chooses with respect to the condition that traversing the path boundary region remains at the left. To reduce the number of nodal points the numerical integration is performed using Sidi sigmoidal non-linear transformation (Sidi, 2006):

$\theta=G(\xi)=\xi-\frac{\sin (2 \pi \xi)}{2 \pi}, 0<\xi<2 \pi$. $g(\theta)$.

Then, at the boundary it holds that $t=\varphi(\theta)+i \cdot \psi(\theta)=$

Solution of integral equations (3) - (4) is performed using the method of mechanical quadratures. For integrals with Cauchytype kernels quadrature formulas of the form (Kolm and Rokhlin, 2001; Eshkuvatov et al., 2009) are used:

$\int_{\Omega} \frac{\mathrm{q}}{\mathrm{t}-\mathrm{z}_{\mathrm{v}}} \mathrm{dt}=\mathrm{h} \sum_{\mathrm{n}=1}^{\mathrm{K}} \mathrm{q}_{\mathrm{n}} \frac{\mathrm{g}_{\mathrm{n}}^{\prime}}{\mathrm{t}_{\mathrm{n}}-\mathrm{z}_{\mathrm{v}}}$,

$t_{n}=g\left(\theta_{n}\right), g_{n}^{\prime}=g^{\prime}\left(\theta_{n}\right), \theta_{n}=n \cdot h, z_{v}=z\left(\tilde{\theta}_{v}\right)$,

$\tilde{\theta}_{v}=\theta_{v}+\frac{h}{2}, \quad v=\overline{1, K}, q_{n}=q\left(t_{n}\right), h=\frac{2 \pi}{K}$.

And for other of the integrals the quadrature formulas of the form (Mikulich, 2012) are applied:

$\int_{\Omega} q \cdot f\left(t, z_{v}\right) d t=h \sum_{n=1}^{K} q_{n} \cdot f_{m} \cdot g_{n}^{\prime}$,

where $f_{m}=f\left(t_{n}, z_{v}\right)$.

Replacing the integrals with the specified quadrature formulas, the system of linear algebraic equations for determination of the nodal values of unknown boundary functions $q^{R}$ and $q^{I}$ is obtained:

$\bar{q}_{v}^{R}+h \sum_{n=1}^{K} f_{1 m n}^{R} q_{n}^{R} g_{n}^{\prime}+h \sum_{n=1}^{K} f_{2 \downarrow n}^{R} \bar{q}_{n}^{R} \bar{g}_{n}^{\prime}-h \sum_{n=1}^{K} f_{1 \downarrow n}^{I} q_{n}^{I} g^{\prime}{ }_{n}$ $-h \sum_{n=1}^{K} f_{2 m}^{I} \bar{q}_{n}^{I} \bar{g}_{n}^{\prime}=2 S_{v}^{R}$,

$\bar{q}_{v}^{I}+h \sum_{n=1}^{K} f_{1 m}^{R} q_{n}^{I} g^{\prime}{ }_{n}+h \sum_{n=1}^{K} f_{2 m}^{R} \bar{q}_{n}^{I} \bar{g}_{n}^{\prime}+h \sum_{n=1}^{K} f_{1 m}^{I} q_{n}^{R} g^{\prime}{ }_{n}$ $+h \sum_{n=1}^{K} f_{2 m}^{I} \bar{q}_{n}^{R} \bar{g}_{n}^{\prime}=2 S_{v}^{I}$ where $\bar{q}_{v}^{R}=\bar{q}^{R}\left(z_{v}\right), q_{n}^{R}=q^{R}\left(t_{n}\right), \quad \bar{q}_{v}^{I}=\bar{q}^{I}\left(z_{v}\right)$, are real and imaginary parts of the unknown functions at the boundary, $f_{i v n}^{R}=$ $f_{i}^{R}\left(t_{n}, z_{v}\right), f_{i v n}^{I}=f_{i}^{I}\left(t_{n}, z_{v}\right), i=1,2, S_{v}^{R}=S^{R}\left(z_{v}\right)$, $S_{v}^{I}=\mathrm{S}^{\mathrm{I}}\left(\mathrm{z}_{\mathrm{v}}\right)$ are known function.

Calculations were performed in the absence of contact of boundary of incisions that was tested on the basis of Esq. (5)(6).

After determination of the unknown functions, stress state of the plate is calculated by dependencies, which are obtained in accordance with representation (1) by providing singular components in the kernels of equations and consequently using Plemelj-Sokhotski formulas:

$2\left(\sigma_{\theta_{v}}-i \tau_{s \theta_{v}}\right)=2\left(\sigma_{\theta_{v}}^{R}-i \tau_{s \theta_{v}}^{R}\right)+2 i\left(\sigma_{\theta_{v}}^{I}-i \tau_{s \theta_{v}}^{I}\right)$,

$2\left(\sigma_{\theta_{v}}^{R}-i \tau_{s \theta_{v}}^{R}\right)=\frac{1+v}{2} q_{v}^{R}+\frac{1-v}{2} \bar{q}_{v}^{R}+h \sum_{n=1}^{K} \tilde{f}_{1 m}^{R} q_{n}^{R} g^{\prime}{ }_{n}$ $+h \sum_{n=1}^{K} \tilde{f}_{2 m}^{R} \bar{q}_{n}^{R} \bar{g}_{n}^{\prime}-h \sum_{n=1}^{K} \tilde{f}_{1 m}^{I} q_{n}^{I} g^{\prime}{ }_{n}-$ $h \sum_{n=1}^{K} \tilde{f}_{2 m}^{I} \bar{q}_{n}^{I} \bar{g}_{n}^{\prime}+\widetilde{\Phi}_{v}^{R}$,

$2\left(\sigma_{\theta_{v}}^{I}-i \tau_{s \theta_{v}}^{I}\right)=\frac{1+v}{2} q_{v}^{I}+\frac{1-v}{2} \bar{q}_{v}^{I}+h \sum_{n=1}^{K} \tilde{f}_{1 m}^{R} q_{n}^{I} g^{\prime}{ }_{n}$ $+h \sum_{n=1}^{K} \tilde{f}_{2 m}^{R} \bar{q}_{n}^{I} \bar{g}_{n}^{\prime}+h \sum_{n=1}^{K} \tilde{f}_{1 m}^{I} q_{n}^{R} g^{\prime}{ }_{n}+$ $h \sum_{n=1}^{K} \tilde{f}_{2 m}^{I} \bar{q}_{n}^{R} \bar{g}_{n}^{\prime}+\widetilde{\Phi}_{v}^{I}$

where $\quad \tilde{f}_{i v n}^{R}=\tilde{f}_{i}^{R}\left(t_{n}, z_{v}\right), \tilde{f}_{i v n}^{I}=\tilde{f}_{i}^{I}\left(t_{n}, z_{v}\right) ; \quad i=1,2 ; \widetilde{\Phi}_{v}^{R}$, $\widetilde{\Phi}_{v}^{\mathrm{I}}$ are the values of known functions in selected points of collocation, which are obtained similar to Mikulich and Maksymovych (2011).

\subsection{Numeric calculation stresses in the plate}

Based on the developed technique the distribution of maximal stresses in the plate with an incision under the actions of oscillating forces $Q_{1} e^{i \omega \tau}, Q_{2} e^{i \omega \tau}$ is studied. The forces are applied at the points $(0 ; \pm b)$.

The results of calculations of dynamic stresses are attributed to the intensity of the stresses oscillating forces.

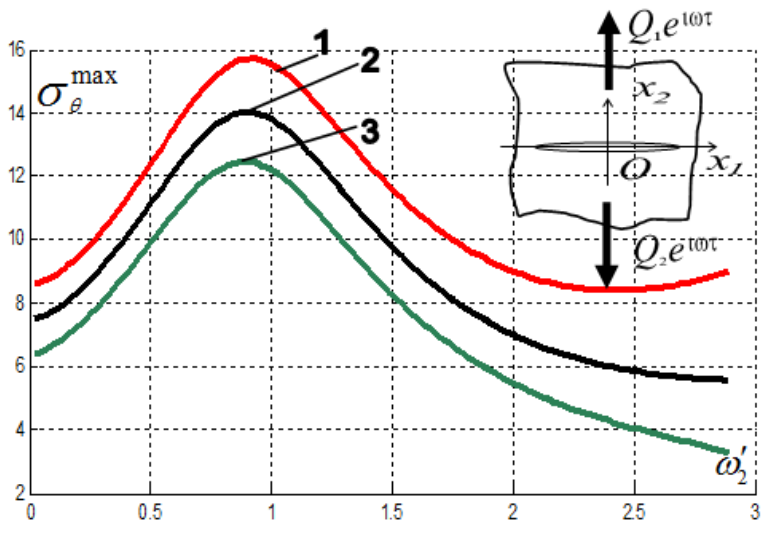

Fig. 2. Maximum dynamic stresses in the plate with a horizontal incision

Fig. 2 shows the results of numerical calculation of the dependence of the maximum dynamic stresses on the dimensionless frequency $\omega_{2}^{\prime}=\frac{\omega \cdot a}{c_{2}}$ of the applied forces, where $c_{2}$ is the speed of shear waves. The incision in the plate is modeled as an elliptic hole with axis ratio of 10 . Calculations were performed for different values of the distance between the point of application of 
the oscillating forces and the center of the incision. The calculations were performed for 200 nodal points at the boundary of the incision. The Poisson's ratio was equal to 0.3. In Fig. 2 curve 1 corresponds to the case of $b=a$; curve 2 corresponds to the case of $b=1.5 a$ and curve 3 corresponds to the case of $b=$ $2 a$, where $a$ is a major semi axis of the incision.

Fig. 2 shows that the maximum dynamic stresses have the fluctuating nature. At high frequencies, a significant increase in the stresses doesn't occur due to the absence of contact at the boundary of incision.

Analysis of the numerical results shows that the maximum stresses at the boundary of the incision are increasing (for a range of frequencies in $(0.01,0.9))$, and then they decrease and become lower comparing to those under static loads.

The maximum dynamic stresses exceed the corresponding static ones in 1.82 times for the case, when the distance to the points of application of forces is equal to the major semi-axis of the incision. With the increase in distance to the point of application of forces the maximum dynamic stresses exceed static in 1.86 times for $b=1.5 a$ and in 1.93 times for $b=2 a$.

Values of dynamic stresses at the boundary of incision at specific values of the frequency for oscillating concentrated forces $Q_{1} e^{i \omega \tau}, Q_{2} e^{i \omega \tau}$, which are applied at the points $(0, a)$ and $(0,-a)$ and Poisson ratio of 0.3 are determined. The results are shown in Fig. 3. Here $\theta$ is the angle in radians.

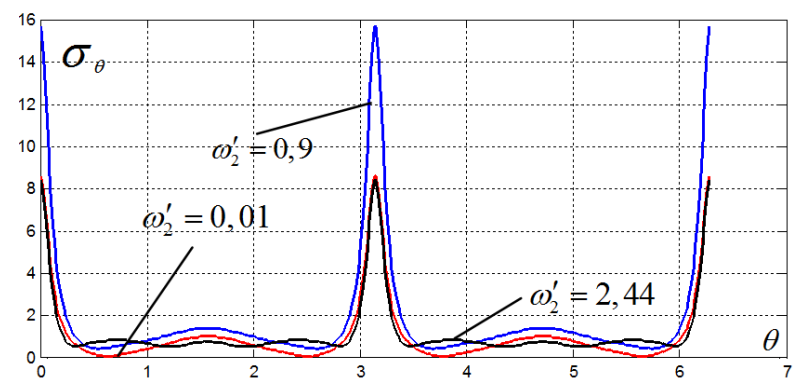

Fig. 3. Distributions of stresses on the boundary of the horizontal incision

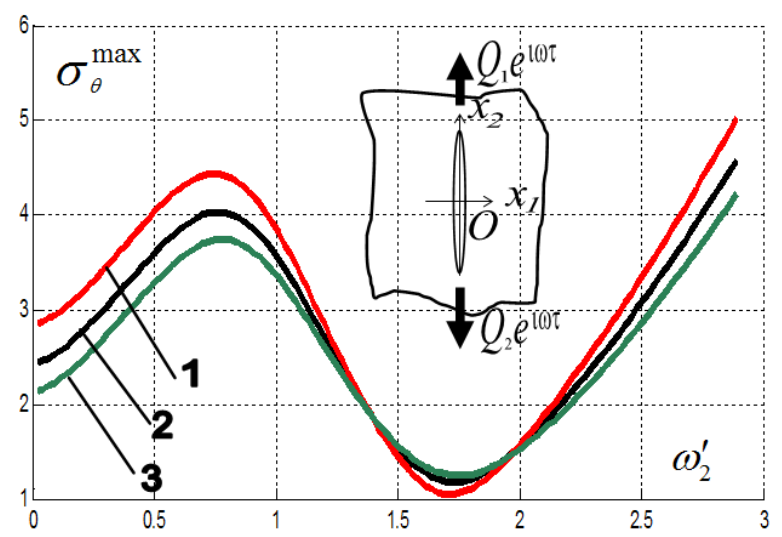

Fig. 4. Maximum dynamic stresses in the plate with a vertical incision

The figure shows that the maximum stresses are occurring at the end of the major semi-axis. There is no significant change in the distribution of stresses along the boundary of incision with increasing in frequency.

The effect of orientation of incision on the distribution of maximum dynamic stresses is also studied. The values of the maximum stresses in the plate with a vertical incision for different values of dimensionless frequency $\omega_{2}^{\prime}=\frac{\omega \cdot a}{c_{2}}$ are calculated. The results are shown in Fig. 4.

Calculations were performed for different values of the distance from the point of application of the oscillating forces to the center of the incision. In numerical calculations 250 nodal points meshed the boundary of the incision, and the Poisson ratio was equal to 0.3. In Fig. 4 curve 1 corresponds to the case of $b=$ $1.8 a$; curve 2 corresponds to the case of $b=2 a$ curve 3 corresponds to the case of $b=2.2 a$, where $a$ is the major semi-axis of the incision.

Fig. 2 shows that the maximum dynamic stresses have the fluctuating nature.

The maximum stresses are observed at the frequency of 0.75 , and minimum stresses occur at frequency of 1.72 . Increase in the frequency of the applied oscillating force causes a significant increase in stresses, which exceed the static ones.

The maximum dynamic stresses exceed the corresponding static in 1.55 times for the case when the distance to the points of application of forces is $b=1.8 a$. With increase in distance to the point of application of forces the maximum dynamic stresses exceed static in 1.65 times for $b=2 a$ and in 1.75 times for $b=$ $2.2 a$.

Values of dynamic stresses at the boundary of vertical incision at specific values of the frequency for oscillating concentrated forces $Q_{1} e^{i \omega \tau}, Q_{2} e^{i \omega \tau}$, which are applied at the points $(0,1.8 a)$ and $(0,-1.8 a)$ and Poisson's ratio of 0.3 are determined. The results are shown in Fig.5. Here $\theta$ is the angle in radians.

The figure shows that the maximum stress occurs at the end of the major semi-axis. With increase in frequency the decrease in the oscillating nature of the distribution of stresses along the incision boundary is observed.

With increase in frequency of the applied load stress distribution along the boundary of the incision changes. Therefore, to study the dynamic stress state it is not enough to determine the value of stress just at a few points. This demonstrates the significant accuracy of the proposed algorithm as opposed to the methods of series and boundary shape perturbation. Since these methods define stresses only at specific points, but not along the boundary.

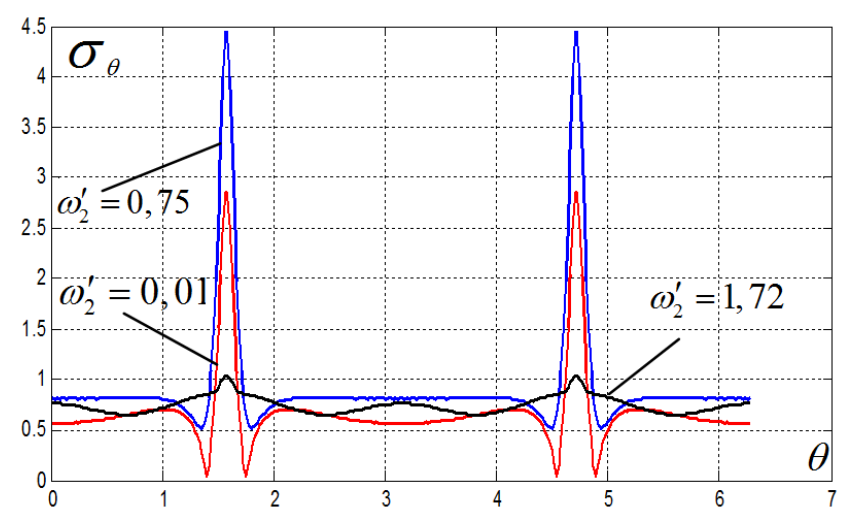

Fig. 5. Distributions of stresses on the boundary of the vertical incision

The dependence of the distribution of maximum dynamic stress from the inclination angle of the incision is investigated. Concentrated forces $Q_{1} e^{i \omega \tau}, Q_{2} e^{i \omega \tau}$ are applied at the points $(0,2 a)$ and $(0,-2 a)$. The calculations were performed for 250 
nodal points at the boundary of the incision and the Poisson's ratio of 0.3 . In fig. 6 curve 1 corresponds to the case of $0^{\circ}$, curve $2-30^{\circ}$, curve $3-45^{\circ}$, curve $4-60^{\circ}$, curve $5-90^{\circ}$, where is an angle between the major axis of the incision and $O x_{1}$. axis.

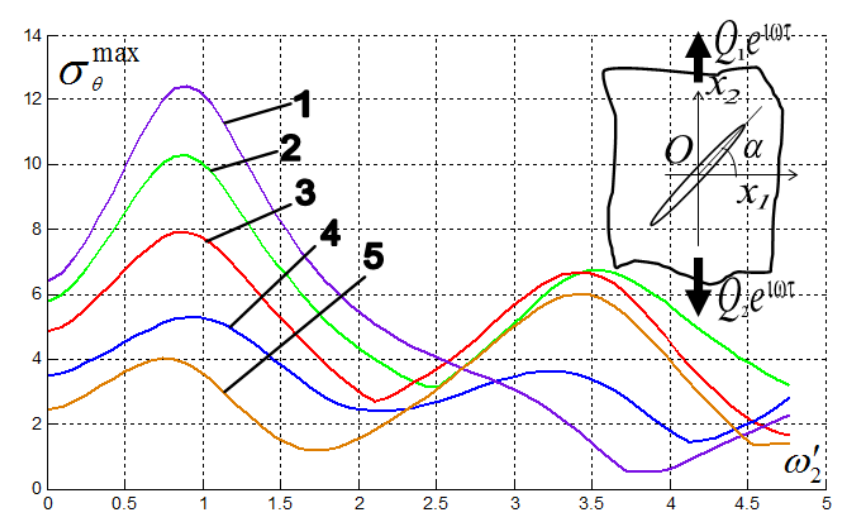

Fig. 6. Maximum dynamic stresses in the plate with an incision

Fig. 6 shows that the maximal dynamic stresses have the fluctuating nature regardless of the inclination of the incision. The maximum dynamic stresses exceed the corresponding static for the case when the frequency $\omega_{2}^{\prime}$ of the applied load is in the range $(0.9 ; 1)$. For vertical incision maximum dynamic stresses occur at a frequency $\omega_{2}^{\prime}=3.45$.

\section{SUMMARY}

The technique developed in this paper allows to study the stresses at the boundary of incisions in plates under the action of concentrated oscillating forces. Effects of orientation of incisions on the stress distribution are studied. Effects of the distance between the incisions on the stress distribution are investigated.

The advantage of the proposed algorithm is the ability of determination of the dynamic stresses along the entire boundary, and not at the only specific point. This makes it possible to investigate in details the dynamic stress state of defective plates.

\section{REFERENCES}

1. Banerjee P. (1994) Boundary element method in engineering science, McGraw Hill, New York, London.

2. Bonnet M. (1995), Integral equations and boundary elements. Mechanical application of solids and fluids (in French), CNRS Éditions / Éditions EYROLLES, Paris.

3. Brebbia C., Telles J., Wrobel L. (1984), Boundary element techniques, Springer, New York.

4. Elbert Á., Laforgia A. (1986), Monotonicity properties of the zeros of Bessel functions, SIAM Journal on Mathematical Analysis, 17, 1483-1488.

5. Eshkuvatov Z. K., Nik Long N. M. A., Abdulkawi M. (2009), Quadrature formula for approximating the singular integral of Cauchy type with unbounded weight function on the edges, Journal of Computational and Applied Mathematics, 233, 334-345.

6. Guz A.M. Kubenko V., Chernenko M. (1978) The diffraction of elastic waves, Naukova Dumka, Kyiv.

7. Kolm P., Rokhlin V. (2001), Numerical Quadratures for Singular and Hypersingular Integrals, Computers and Mathematics with Applications, 41, 327-352.

8. Kubenko V. (1967) Dynamic stress concentration around an elliptical hole, Reports of the Academy of Sciences USSR, 3, 60-64.

9. Kupradze V. (1963) Methods of potential in the theory of elasticity, Fizmatgiz, Moscow.

10. Mikulich O. A. (2012), Stress state of plate elements with rigid inclusion of arbitrary shape at a steady-state oscillations, Naukovi notatky, 39, 118-123.

11. Mikulich O. A., Maksymovych V. M. (2011), Study of interaction holes in infinity plates at a steady-state oscillations, Naukovi notatky, 33, 164-169.

12. Mow C., Mente L. (1963): Dynamic stresses and displacements around cylindrical discontinuities due to plane harmonic shear waves, Journal of Applied Mechanics, 30, 598-604.

13. Mushelishvili N.(1966) Some selected problems of mathematical theory of elasticity, Moscow.

14. Panasyuk V., Savruk M., Nazarchuk Z. (1984) The method of singular integral equations in two-dimensional diffraction problems, Naukova Dumka, Kyiv.

15. Pao Y., Mow C. (1971) Diffraction of elastic waves and dynamic stress concentration, Crane Russak, New York.

16. Savin G. N. (1968), Distribution of the stresses near the holes, Naukova Dumka, Kyiv.

17. Sherman D. (1962) The method of integral equations in the plane and spatial problems of static elasticity theory, Proceedings of the All-Union Congress on Theoretical and Applied Mechanics, 405-467.

18. Sidi A. (2006), Extension of a class of periodizing variable transformations for numerical integration, Mathematics of Computation, 75(253), 327-343.

19. Sladek J., Sladek V., Atluri S. N. (2000), Local boundary integral equation method for solving problem of elasticity with nonhomogeneous material properties, Computational mechanics, 24, 456-462.

20. Timoshenko S. (1967) Fluctuations in engineering, Nauka, Moscow. 